\title{
Analyser et évaluer la relation entre doctorant et directeur de thèse
}

Pascale Haag $^{1}$

\begin{abstract}
[Résumé] La relation entre doctorant et directeur de thèse constitue l'un des principaux déterminants de la réussite du parcours doctoral. Elle est encore peu étudiée, et les instruments de mesure permettant de l'évaluer demeurent peu nombreux dans le champ de la psychologie. Le présent article présente une synthèse des instruments de mesure qui existent aujourd'hui, accompagnée d'une introduction au cadre théorique dans lequel a été construit chacun d'entre eux : Advisory working alliance inventory (AWAI-S) (Schlosser, Gelso, 2001); Graduate Studies Supervision Scale - Student Perception (G3S-SP) (Bravo, Saint-Mieux, Dubois, 2007); Questionnaire on supervisordoctoral student interaction (QSDI) (Mainhard, van der Rijst, van Tartwijk, Wubbels, 2009) ; Échelle de mesure du soutien aux besoins fondamentaux (Litalien, Guay, 2015). Des pistes de recherche pour l'avenir faisant usage de tels questionnaires sont proposées.

Mots-clés : Doctorat, enseignement supérieur, relation d'encadrement, formation à la recherche.
\end{abstract}

[Abstract] The relationship between doctoral students and thesis supervisors is one of the most decisive factors in the successful completion of PhD programs. There is limited research on the topic, and the measuring instruments to evaluate it are few in psychology. This article reviews the current existing measuring instruments and introduces their theoretical framework: AWAI-S (Schlosser, Gelso, 2001); Graduate Studies Supervision Scale - Student Perception (G3S-SP) (Bravo, Saint-Mieux, Dubois, 2007); Questionnaire on supervisor-doctoral student interaction (QSDI) (Mainhard, van der Rijst, van Tartwijk, Wubbels, 2009); Support Measurement Scale for Basic Needs (Litalien, Guay, 2015). Future research developments using such questionnaires are proposed.

Keywords: PhD, higher education, menthorship, research training.

La relation entre doctorant et directeur de thèse est désormais reconnue comme l'un des principaux déterminants de la réussite du diplôme, tandis qu'une mauvaise relation est souvent associée à un abandon en cours de cursus (Bair, Haworth, 2005 ; Buckley, Hooley, 1998 ; Gelso, 1993 ; 2013 ; Gelso, Lent, 2000 ; Golde, 2005 ; Lovitts, 2001 ; Tinto, 1993 ; Vaccaro, 2009). La proportion de décrochage au cours du doctorat est importante $-40 \%$ à $50 \%$ en Amérique du Nord (Litalien, Guay, 2015), entre 10 et $30 \%$ dans les disciplines scientifiques et jusqu'à $60 \%$ dans les sciences sociales (Moguérou, Murdoch, Paul, 2003), et ses conséquences sont importantes : sur le plan de l'emploi et de l'estime de soi pour les doctorants qui n'achèvent pas le cursus (Lovitts, 2001 ; Litalien, Guay, 2015) autant que sur le plan économique pour l'université, compte tenu du

1 École des hautes études en sciences sociales, Paris. 
temps investi par les enseignants et des coûts associés aux années d'études (Wendler et al., 2012).

La relation d'encadrement a été analysée du point de vue quantitatif, à travers des questionnaires visant à évaluer le degré de satisfaction du doctorant par rapport à son directeur de thèse (par exemple Hasksever, Manisali, 2000 ; Heath, 2002) aussi bien que qualitatif, à la lumière d'entretiens réalisés avec des doctorants afin de mieux comprendre l'expérience qu'ils font de cette relation (Golde, 2000 ; Zuber-Skerrit, Roche, 2004). Les travaux s'intéressant au point de vue de l'encadrant sont moins nombreux (Goodmann, 2006; Knox et al., 2011), et l'absence de formation à l'encadrement est fréquemment soulignée dans la littérature (e.g. Amundsen, McAlpine, 2009 ; Bravo, 2007; Emilsson, Johnsson, 2007 ; McAlpine, Amundsen, 2011 ; Voyer, 1992). Force est de constater que la formation à la direction de recherche se fait généralement « sur le tas », par le biais d'échanges entre pairs (Amundsen, McAlpine, 2009) et - en fonction des expériences vécues comme étudiant - en s'attachant à reproduire ou, au contraire, à se différencier de l'encadrement que l'on a connu (Grant, Graham, 1999). Une absence de consensus sur ce qui relève ou ne relève pas de la direction de recherche en résulte, qui se traduit par un large éventail de pratiques qui varient au gré des représentations (Golde, 2007; Hermer, 2012).

Ainsi que le souligne Gérard (2014), les « recherches montrent que l'approche des directeurs de recherche (leur pratique) ne correspond pas toujours aux attentes des jeunes chercheurs ». Il est possible d'inverser la proposition : il n'est pas certain que les doctorants répondent toujours aux attentes de leur directeur de recherche. La difficulté provient principalement de l'absence de communication au sujet des attentes entre les deux protagonistes de cette relation complexe où l'implicite règne bien souvent en maître. Les décalages quant à la perception des rôles respectifs peuvent s'analyser selon trois dimensions (Gérard, 2014) : scientifique (aide théorique, méthodologique, rédactionnelle apportée par le directeur de recherche) ; institutionnelle (construction d'un planning de travail et de recherche, délais de rétroaction) et relationnelle (qualité de la relation interpersonnelle, modalités verbales et non verbales des échanges). Pourtant, les modalités de la relation font rarement l'objet d'un échange explicite : le candidat à la recherche d'un directeur de thèse ose rarement aborder la question de façon directe, et il est rare que l'encadrant pressenti le fasse.

Alors que le doctorat, à l'instar de l'ensemble de l'enseignement supérieur, est en pleine mutation (Matos, 2013; Gorga, Leresche, 2015), alors que la question du devenir des jeunes docteurs se pose de manière toujours plus aigüe (APEC, 2015), le manque d'outils validés et de cadres théoriques solides pour étudier et mieux appréhender la relation d'encadrement est régulièrement souligné (Bravo, 2007; Mainhard et al., 2009; Schlosser, Gelso, 2001). Par ailleurs, les différentes équipes qui s'intéressent à cette question semblent avoir travaillé de manière totalement indépendante, en s'ignorant les uns les autres, sans jamais se citer. 
Le présent article présente une synthèse des instruments de mesure identifiés au cours de cette recherche. Ils sont au nombre de quatre. À ma connaissance, ce sont les seuls qui ont été publiés à ce jour en psychologie. Les quatre premières sections présentent les différents questionnaires : chaque section comprend d'abord une introduction au cadre théorique dans lequel a été construit le questionnaire, puis un descriptif de l'outil lui-même. La cinquième partie suggère des pistes de recherche pour l'avenir en utilisant ces questionnaires.

\section{L'alliance de travail}

\subsection{Alliance thérapeutique et alliance de travail}

Le concept d'alliance de travail trouve son origine dans le cadre de la pensée psychanalytique relative à la relation thérapeutique (Etchegoyen, 2005). Il se développe dans le courant de l'ego-psychologie, à partir de Sterba (1929). En 1937, Edward Bibring propose le concept d'une alliance entre le thérapeute et la "part raisonnable de l'ego du patient », tandis que la création du syntagme "alliance thérapeutique » est attribuée à Elisabeth Zetzel (1956), qui insiste sur l'importance de cette composante relationnelle, confiante et stable, dans l'efficacité de la cure. En dehors du contexte psychanalytique également, la force de la relation collaborative est reconnue comme cruciale, la plupart des théoriciens soulignant qu'il est important d'établir une relation entre soignant et soigné, qui constitue un premier pas, essentiel, quelle que soit la méthode thérapeutique (Summers, Barber, 2010).

L'alliance se définit notamment à partir de deux composantes : une dimension personnelle, affective, qui permet d'établir dès les premières séances une confiance et un respect mutuels, et une dimension cognitive qui se rapporte aux buts poursuivis. Selon Green (2009), les qualités du thérapeute (amabilité, confiance, implication) relèvent de la composante personnelle au même titre que certaines caractéristiques du patient (compétences sociales hors du contexte thérapeutique, par exemple). La composante cognitive renvoie au fait que l'objectif du traitement et la méthode sont explicités et font l'objet d'un commun accord.

L'usage du terme « alliance de travail » s'est développé à partir des travaux de Greenson (1967). L'expression a connu une évolution conceptuelle importante inscrivant la conscience, la rationalité et l'objectivité au cœur de cette notion, tandis que son usage s'étendait à d'autres sphères comme l'orientation, l'éducation, le travail social et, finalement, à toutes les relations inductrices de changement (Guillon, 2013). Dans le prolongement des travaux de Greenson, Bordin met en évidence trois composantes, qui seront adaptées par Schlosser et Gelso. Une bonne alliance de travail se traduit par :

- un accord sur les buts poursuivis : une forte alliance de travail se caractérise par une prise en compte et une valorisation commune des objectifs partagés ; 
- un accord sur les tâches ou activités spécifiques à accomplir pour atteindre l'objectif fixé : elles doivent faire l'objet d'un accord entre les membres de la dyade (dimension cognitive);

- la mise en place d'un lien, ce qui renvoie à la dimension affective et émotionnelle de la relation, ainsi qu'à la confiance et à l'acceptation mutuelle.

Guillon (2013) souligne le fait que ces trois composantes, clairement identifiables, sont interdépendantes et que l'alliance de travail est l'expression de leur articulation. Les deux protagonistes de la relation y contribuent, et cette alliance concernant l'accord sur les buts, sur les tâches, et même le lien émotionnel, doit être « consciente, rationnelle et objective ». Force est de constater que, dans le cadre de la relation doctorale, ces dimensions sont rarement explicitées.

\subsection{L'échelle de mesure de l'alliance de travail}

Dans le contexte du doctorat, Schlosser et Gelso (2001:158) définissent l'alliance de travail comme «la portion de la relation qui reflète la connexion entre "conseiller" (advisor) et "conseillé" (advisee), développée au cours du travail en vue d'un but commun ». L'Advisory working alliance inventory (AWAI-S) est un auto-questionnaire conçu pour évaluer la perception qu'a un graduate student de la relation avec son directeur de recherche. Les questions ont été développées à partir du Working Alliance Inventory (Horvath, Greenberg, 1989) et du Supervisory working alliance inventory (Efstation, Patton, Kardash, 1990), complétés par le Research Training Environment Scale-Revised (Gelso, Mallinckrodt, Judge, 1996) et la Research Advisor Functions Scale (Hollingsworth, Fassinger, 2002). Trois facteurs ont été retenus : rapport, task focus et identification. Le questionnaire comprend trente items évalués sur une échelle de type Likert en sept points (c'est-à-dire une échelle de jugement par laquelle la personne interrogée exprime son degré d'accord ou de désaccord vis-à-vis d'un énoncé, de 1 = pas du tout d'accord à 7 = entièrement d'accord).

Dans ce cadre, le rapport renvoie à la composante de soutien et d'encouragement dans la relation de conseil - le conseiller étant ici le directeur de thèse et le conseillé le doctorant ou l'étudiant de master. Le lien émotionnel qui se tisse au cours du travail effectué en commun participe également de cette notion. Cette sous-échelle comprend onze questions, dont huit items inversés, c'est-à-dire formulés de manière négative (par exemple, « Dans nos échanges, mon directeur de thèse est ouvert à mes propositions. » ; " J’ai l'impression que mon directeur de thèse ne m’apprécie pas beaucoup. »).

L'apprentissage correspond à la dimension task focus et désigne la partie de la relation où le conseiller aide à comprendre les tâches, les buts et le processus du parcours doctoral. Elle prend en compte la manière dont le directeur de thèse forme à la recherche, enseigne à faire face aux difficultés et accompagne le développement professionnel. Cet aspect concerne également la capacité de la dyade à rester concentrée sur le travail en cours. Cette sous-échelle comprend quatorze questions, dont trois items inversés 
(p. ex. " Mon directeur de thèse facilite mon insertion professionnelle en utilisant ses réseaux. » ; « Mon directeur de thèse m’aide à reconnaître dans quels domaines je peux progresser. »).

Enfin, l'identification concerne le désir que peut avoir l'étudiant ou le doctorant de ressembler au conseiller. Cette sous-échelle comprend cinq items inversés (p. ex. « Je ne veux pas avoir l'impression de mener mes travaux de la même manière que mon directeur de thèse. »; "Mon directeur de thèse et moi avons des centres d'intérêts différents. »).

\section{Perception de la qualité de l'encadrement en sciences de la santé}

\subsection{Le manque d'évaluation de la qualité de l'encadrement}

Bravo, Saint-Mieux et Dubois (2007) partent du constat que la supervision d'étudiants est une tâche complexe, à laquelle les enseignants du supérieur sont rarement formés au moment de leur recrutement. Ils soulignent en outre le fait que, contrairement aux activités d'enseignement et de recherche, la supervision d'étudiants est rarement évaluée. Ils ne font pas référence à un cadre théorique spécifique, mais considèrent qu'en dépit de l'existence de travaux sur l'encadrement, il manque encore des outils de mesure fiables et validés pour évaluer cette perception. La seule exception notable serait le Postgraduate Research Experience Questionnaire (Drennan, 2008) développé par le Graduate Careers Council of Australia, mais il porte plus généralement sur l'ensemble du cadre universitaire et ne comporte que quelques questions sur la relation d'encadrement. En conséquence, Bravo et al. (2007) se proposent de combler cette lacune en étudiant la perception qu'ont les étudiants de second cycle (graduate students) de la qualité de leur encadrement, envisagé comme une « relation dyadique entre étudiant et superviseur dans laquelle chacun porte une part de responsabilité par rapport au succès de la relation.»

L'échelle est destinée à permettre d'évaluer périodiquement la qualité de la supervision du point de vue de l'étudiant, de façon à offrir un feedback à l'encadrant sur ses points forts et sur ce qu'il lui reste à améliorer. Les auteurs souhaitent que cet outil puisse servir de base à un échange fructueux entre l'étudiant ou le doctorant et son directeur de recherche, permettant d'aborder aussi bien les sources de satisfaction que les problèmes.

\subsection{La Graduate Studies Supervision Scale - Student Perception (G3S-SP)}

Le Graduate Studies Supervision Scale - Student Perception (G3S-SP) est un questionnaire auto-administré conçu dans le cadre des sciences de la santé pour évaluer la perception qu'ont les étudiants de second et troisième cycle de la qualité de leur supervision. Les 
différents items ont été choisis d'une part en prenant appui sur la littérature scientifique sur la supervision, d'autre part en se fondant sur l'expérience personnelle des auteurs. Afin d'optimiser la validité de contenu, les questions prennent en considération tous les aspects de la relation d'encadrement, de l'accompagnement dans la découverte de l'environnement académique à la publication des résultats.

Le questionnaire comprend dix-sept items évalués sur une échelle de type Likert en quatre points. Deux dimensions sont évaluées : le degré d'implication du directeur de recherche dans le projet du doctorant au moyen de neuf items (p. ex., « Mon directeur m’a aidé(e) à structurer les étapes de ma recherche »; « Depuis mon inscription, mon directeur et moi avons discuté des modalités de notre collaboration. ») et les relations interpersonnelles au moyen de huit items (p. ex., « Je me sens à l'aise d'évoquer avec mon directeur mes insatisfactions à son égard »; « Au plan interpersonnel, j’entretiens une relation harmonieuse avec mon directeur »).

\section{Perception du style interpersonnel du directeur de recherche}

\subsection{L'approche systémique}

Mainhard, van der Rijst, van Tartwijk et Wubbels (2009) se réfèrent aux théories de la communication élaborée par Paul Watzlawick. Ils prennent notamment appui sur l'un des cinq axiomes de la communication de Watzlavick, à savoir, le fait que « toute communication peut s'analyser sous le double aspect du contenu et de la relation » (Watzlawick et al., 1967). L'interaction est définie comme un échange de messages constitués d'une part d'informations (le contenu), de l'autre du comportement relationnel.

Lors d'une interaction régulière, comme celle d'un doctorant et de son directeur de recherche, certains schémas se mettent en place. Ils dépendent des deux protagonistes, le comportement de l'un influençant en retour celui de l'autre. C'est ce qui explique que le style relationnel d'un individu puisse varier en fonction de la personne avec qui elle interagit. Pour décrire le style relationnel du directeur de recherches, les auteurs se sont inspirés des travaux de Wubbels et al. (2006), réalisés à partir du modèle de Leary (1957). Ce dernier propose de décrire les comportements interpersonnels selon un modèle "circomplexe " permettant de représenter des variables qui ont une relation d'ordre circulaire. Les variables sont représentées sur un plan comprenant deux axes orthogonaux : l'influence exercée sur les élèves - ou, ici, les doctorants - (axe vertical) et la distance affective que l'enseignant entretient avec eux (axe horizontal). Ces deux dimensions s'inscrivent dans un continuum qui va, pour la première dimension (" contrôle »), de l'autorité à la subordination et, pour la seconde (" affectivité »), de l'opposition à la coopération, comme l'illustrent la figure et le tableau explicatif (voir fig. 1 et tableau 1). 


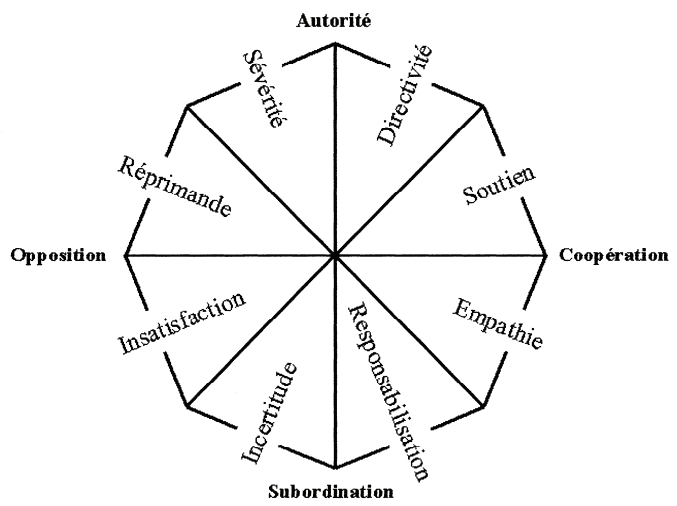

Figure 1. Axes et échelles du Profil interpersonnel du stagiaire (Genoud, 2003)

\begin{tabular}{ll}
\hline ÉCHELLE & EXPLICATION \\
\hline Directivité (AC) & $\begin{array}{l}\text { Dirige, organise clairement, donne des ins- } \\
\text { tructions, a le contrôle, stimule. }\end{array}$ \\
\hline Soutien (CA) & $\begin{array}{l}\text { Aide, montre de l'intérêt pour son docto- } \\
\text { rant, l'encourage. }\end{array}$ \\
\hline Empathie (CS) & $\begin{array}{l}\text { Est compréhensif, se met à la portée de son } \\
\text { doctorant, est patient et ouvert, sait plaisan- } \\
\text { ter. }\end{array}$ \\
\hline Responsabilisation (SC) & $\begin{array}{l}\text { Donne des libertés, des opportunités d'in- } \\
\text { dépendance, fait confiance. }\end{array}$ \\
\hline Incertitude (SO) & $\begin{array}{l}\text { Garde un profil bas, hésite, est inquiet, est } \\
\text { désorganisé. }\end{array}$ \\
\hline Insatisfaction (OS) & $\begin{array}{l}\text { Est insatisfait, mécontent, se méfie du doc- } \\
\text { torant, est de mauvaise humeur. }\end{array}$ \\
\hline Réprimande (OA) & $\begin{array}{l}\text { Exprime de la colère et de l'irritation, pose } \\
\text { des interdits, sanctionne }\end{array}$ \\
\hline Sévérité (AO) & $\begin{array}{l}\text { Tient les rênes serrées, contrôle, réduit au } \\
\text { silence, est strict. }\end{array}$ \\
\hline
\end{tabular}

Tableau 1. Explications des échelles (adapté de Genoud, 2003)

\subsection{Le questionnaire on supervisor-doctoral student interaction (QSDI)}

Le QSDI est destiné à « récolter des informations sur la perception qu'ont les doctorants du style interpersonnel de leur directeur de recherche ». Il est élaboré à partir du Questionnaire on teacher interaction (Wubbels et al., 2006), conçu à l'origine pour évaluer le comportement interactionnel d'enseignant du secondaire et du Questionnaire on Supervisor Interaction (Kremer-Hayon, Wubbels, 1993), utilisé dans le cadre de la supervision 
d'enseignants stagiaires par des enseignants confirmés.

Le QDSI comprend quarante-et-un items, qui mesurent la relation en fonction des deux dimensions inscrites sur les axes autorité/subordination d'une part, opposition/coopération de l'autre, sur une échelle de type Likert en cinq points. Huit styles interactionnels qualifiant le comportement du directeur de recherche sont identifiés : directivité (six items, p. ex. « [...] gives me clear guidance ») always cooperates, if I want something ») ; empathie (quatre items, p. ex. « [...] pays attention, if I have something to say »); responsabilisation (quatre items, p. ex. "[...] lets me choose my own direction ») ; incertitude (six items, p. ex. « [...] is unclear during our conversations »); insatisfaction (six items, p. ex. " [...] says that I am unskilled »); réprimande (quatre items, p. ex. « [...] humiliates me »); sévérité (cinq items, p. ex. « [...] is quick to criticize $m e$ ».

\section{Mesure du soutien aux besoins psychologiques}

\subsection{La théorie des besoins psychologiques fondamentaux}

La théorie des besoins psychologiques fondamentaux s'inscrit dans le cadre plus large de la théorie de l'autodétermination (Deci, Ryan 2000). Ils proposent en particulier une théorie du bien-être psychologique fondée sur le postulat que les êtres humains ont des besoins psychologiques fondamentaux. Ils définissent ces besoins comme des «nutriments psychologiques innés essentiels pour le développement psychologique, l'intégrité et le bien-être » (Deci, Ryan, 2000). La satisfaction de ces derniers constitue le mécanisme essentiel qui détermine la santé psychologique et le fonctionnement optimal des individus.

Le besoin d'autonomie de l'individu est satisfait lorsque celui-ci se sent libre de choisir et d'organiser lui-même ses actions (Deci, Ryan, 2000). L'autonomie renvoie au fait qu'une personne dispose d'une latitude décisionnelle suffisante pour se percevoir comme l'agent qui réalise une action donnée (De Charms, 1968; Deci, Ryan, 1985). L'autonomie est conçue comme un continuum allant d'un comportement totalement contrôlé par autrui à un comportement totalement autonome (Deci, Ryan, 2000).

La notion de compétence fait référence au sentiment d'efficacité (Deci, 1975; White, 1959). Il est comblé lorsque l'individu se sent apte à exécuter une tâche et atteint les résultats escomptés (Deci, Ryan, 2000), mais aussi lorsque la réalisation de la tâche s'accompagne d'un retour constructif (Vallerand, Reid, 1984; Deci, Vallerand, Pelletier, Ryan, 1991).

${ }^{2}$ Dans la mesure où le QSDI est en anglais, les exemples sont ici dans la langue originale. 
Le sentiment d'appartenance sociale (relatedness) implique de développer avec autrui des relations satisfaisantes et sécurisantes, caractérisées par le respect mutuel. Le besoin d'appartenance sociale est complémentaire de celui d'autonomie (Baumeister, Leary, 1995 ; Deci, Ryan, 2000).

La satisfaction des besoins psychologiques fondamentaux définit le minimum requis pour qu'une personne soit en bonne santé tout en informant sur ce que l'environnement doit fournir pour qu'elle se développe et grandisse psychologiquement dans les meilleures conditions possibles. Cette théorie explique donc les conditions sous lesquelles le bien-être d'un individu est facilité au lieu d'être entravé. En un mot, elle propose une psychologie sociale du bien-être psychologique (Ryan, 1995).

Les travaux prenant pour cadre la théorie des besoins psychologique sont nombreux, mais ils portent rarement sur les doctorants. Seules deux thèses canadiennes réalisées à vingt ans d'intervalle sont consacrées aux liens entre la motivation et la persistance dans le cursus doctoral (Losier, 1994 ; Litalien, Guay, 2015), tandis que les liens entre encadrement et besoin d'autonomie ont été étudiés par Overall, Deane, Peterson (2011).

\subsection{L'échelle de mesure du soutien aux besoins fondamentaux}

À partir de trois échelles différentes (Connell, Wellborn, 1991 ; Williams, Deci, 1996 ; Markland, Tobin, 2010), Litalien et Guay (2015) ont créé un instrument de mesure destiné à évaluer la perception qu'ont les doctorants du soutien offert par le directeur de thèse, par d'autres enseignants et chercheurs du laboratoire ou de l'université et par les pairs. Seule l'échelle concernant le directeur de thèse est présentée ici. Elle comprend vingt-sept items qui mesurent la qualité du soutien aux trois besoins fondamentaux définis par la théorie de l'autodétermination: soutien à l'autonomie, en sept items (p. ex. « En général, mon directeur de thèse m’encourage à formuler mes propres idées » $\mathrm{ou}$ « Mon directeur de thèse me permet d'organiser mes études à ma guise, selon mes choix et mes intérêts »), soutien au sentiment de compétence, en douze items (p. ex. "Mon directeur de thèse me donne confiance en mes capacités à réussir mon doctorat » $\mathrm{ou}$ « Mon directeur de thèse est clair et précis dans ses rétroactions et ses réponses à mes questions ») et soutien au sentiment d'affiliation sociale, en huit items (p. ex. « Mon directeur de thèse semble m'apprécier » ou « Mon directeur de thèse s’intéresse à ce que je fais en dehors de la sphère universitaire »).

Dans la mesure où les corrélations étaient très fortes entre les différentes dimensions de l'échelle (entre $r=.75$ et $r=.90$ ), ce qui est mesuré est uniquement un score général de soutien aux besoins fondamentaux.

\section{Synthèse et perspectives de recherches}

Le tableau synoptique qui suit offre une vue d'ensemble des instruments présentés : 


\begin{tabular}{|c|c|c|c|c|}
\hline & $\begin{array}{l}\text { AWAI (Schlos- } \\
\text { ser, Gelso, } \\
\text { 2001) }\end{array}$ & $\begin{array}{l}\text { G3S-SP (Bravo, } \\
\text { Saint-Mleux, Du- } \\
\text { bois, 2007) }\end{array}$ & $\begin{array}{l}\text { QSDI (Mainhard, } \\
\text { v. der Rijst, Tart- } \\
\text { wijk, Wubbels, } \\
\text { 2009) }\end{array}$ & $\begin{array}{l}\text { SPN (Litalien, } \\
\text { Guay, 2014) }\end{array}$ \\
\hline $\begin{array}{l}\text { But de } \\
\text { l'outil }\end{array}$ & $\begin{array}{l}\text { Évaluer la re- } \\
\text { lation d'enca- } \\
\text { drement du } \\
\text { point de vue } \\
\text { du doctorant }\end{array}$ & $\begin{array}{l}\text { Évaluer la per- } \\
\text { ception par le } \\
\text { doctorant de la } \\
\text { qualité de leur } \\
\text { encadrement }\end{array}$ & $\begin{array}{l}\text { Évaluer la percep- } \\
\text { tion par le docto- } \\
\text { rant du style inter- } \\
\text { personnel de son } \\
\text { directeur de re- } \\
\text { cherche }\end{array}$ & $\begin{array}{l}\text { Évaluer la qualité } \\
\text { du soutien aux } \\
\text { besoins psycholo- } \\
\text { giques par le di- } \\
\text { recteur de re- } \\
\text { cherche }\end{array}$ \\
\hline $\begin{array}{l}\text { Cadre } \\
\text { théorique }\end{array}$ & $\begin{array}{c}\text { Alliance de } \\
\text { travail }\end{array}$ & - & $\begin{array}{c}\text { Théorie systé- } \\
\text { mique de la com- } \\
\text { munication }\end{array}$ & $\begin{array}{c}\text { Théorie de } \\
\text { l'auto-détermi- } \\
\text { nation }\end{array}$ \\
\hline Sources & $\begin{array}{c}\text { SWAI, RTES-R } \\
\text { et RAFS }\end{array}$ & $\begin{array}{l}\text { PREQ et focus } \\
\text { groupes }\end{array}$ & QTI et PREQ & RAPS, LCQ et NS \\
\hline $\begin{array}{l}\text { Nombre } \\
\text { d'items }\end{array}$ & 30 & 17 & 41 & 27 \\
\hline $\begin{array}{l}\text { Nombre } \\
\text { de dimen- } \\
\text { sions }\end{array}$ & $\begin{array}{l}3 \text { (rapport, ap- } \\
\text { prentissage, } \\
\text { identification/ } \\
\text { individuation) }\end{array}$ & $\begin{array}{l}2 \text { (degré d'impli- } \\
\text { cation du direc- } \\
\text { teur de re- } \\
\text { cherche, } \\
\text { relations inter- } \\
\text { personnelles) }\end{array}$ & $\begin{array}{c}2 \text { (coopération, } \\
\text { autorité) }\end{array}$ & $\begin{array}{l}1 \text { (score globale } \\
\text { de soutien à } \\
\text { l'autonomie, la } \\
\text { compétence et } \\
\text { l'appartenance }\end{array}$ \\
\hline
\end{tabular}

Tableau 2. Comparaison entre les quatre instruments de mesure

Ces instruments peuvent être utilisés de différentes manières : dans des recherches, associés à d'autres questionnaires, mais aussi comme outil de « diagnostic » visant d'une part à permettre au doctorant de mieux cerner ses propres attentes par rapport à l'encadrant, d'autre part, à ouvrir un dialogue destiné à favoriser l'ajustement mutuel à l'intérieur du binôme. Enfin, la pratique d'une évaluation systématique de la relation d'encadrement pourrait être encouragée sur la base de questionnaires comme ceux qui sont présentés ici.

\subsection{Pistes de recherches futures}

Ces différents outils semblent avoir été peu utilisés après leur construction et leur validation, que ce soit par leurs auteurs ou par d'autres chercheurs. Pourtant, chaque article s'achève sur des propositions de travaux ultérieurs, dont les thématiques se recouvrent parfois d'une étude à l'autre. En voici un aperçu :

- étudier le lien entre la relation d'encadrement et d'autres variables, comme p. ex. la situation personnelle et familiale, les perspectives de carrière et les aspirations professionnelles (Litalien, Guay, 2015) ou la productivité scientifique (Schlosser, Gelso 2001), les liens entre les styles de supervision et la 
durée du doctorat, la satisfaction des étudiants, ou la qualité de la thèse (Mainhard et al., 2009) ou encore, le rôle d'autres acteurs (pairs, autres chercheurs, techniciens) dans la perception de la qualité de l'encadrement (Bravo et al., 2007);

- étendre les travaux à d'autres échantillons de doctorants - en variant aussi bien les disciplines que les pays, de façon à avoir une meilleure connaissance des différentes cultures universitaires (Bravo et al., 2007 ; Schlosser, Gelso, 2001);

- créer des outils pour étudier également la relation du point de vue du directeur de recherche, de façon à mieux appréhender les dynamiques relationnelles (Mainhard et al., 2009) ;

- ouvrir la voie à des études longitudinales. Litalien et Guay (2015) soulignent ainsi, dans les limitations de leur travail, le fait qu'il s'agisse d'une étude rétrospective et suggèrent de mener des recherches sur l'ensemble de la durée de la thèse, en collaboration avec des universités.

\subsection{Expliciter la relation d'encadrement}

Si la relation entre le doctorant et le directeur de thèse constitue le «noyau dur » de la formation à la recherche, les modalités de cette relation font rarement l'objet d'un échange explicite entre les protagonistes. Il n'est pas forcément évident pour le doctorant de faire état de ses besoins ou de ses attentes en matière de formation, en raison du caractère dissymétrique de la relation, et ce, d'autant plus que le directeur de recherche aura également un rôle particulier à jouer à la fin du parcours doctoral, lors de la soutenance et dans l'accompagnement du développement professionnel qui s'ensuit. C'est pourquoi certains questionnaires ont été conçus de façon à pouvoir servir de support à une évaluation périodique de la qualité perçue de l'encadrement. Ils peuvent donner lieu à une discussion sur les éléments positifs et ceux qui sont à améliorer, ou fournir à l'encadrant un feedback lui permettant de s'améliorer de manière générale et de mieux s'adapter aux besoins spécifiques de chaque doctorant.

C'est dans cet esprit qu'ont travaillé Bravo, Saint-Mleux et Dubois (2007). Alors que les encadrants se sont montrés enthousiastes à l'idée d'utiliser cet instrument pour discuter de la perception de la relation, les participants ont exprimé des doutes quant à la possibilité de l'utiliser dans les situations conflictuelles : la majorité d'entre eux ne pensaient pas qu'un tel questionnaire permettrait d'exprimer ouvertement leur mécontentement, le cas échéant, et certains ont même souligné le risque qu'une discussion ouverte ne fasse qu'empirer les choses et ont affirmé qu'ils préféraient «souffrir en silence ». 


\subsection{Le point de vue des directeurs de recherche}

Les représentations qu'ont les responsables d'encadrement doctoral de cette relation sont, elles aussi, encore peu étudiées. La préparation des doctorants à mener une recherche doctorale, l'enthousiasme, la compatibilité relationnelle, le bénéfice que peut retirer l'encadrant du point de vue intellectuel ou en termes de publications comptent parmi les facteurs qui peuvent jouer un rôle dans la qualité perçue de la relation (Knox et al., 2011). Mieux les connaître, dans différentes disciplines, serait de nature à favoriser un meilleur ajustement de la dyade, dans la mesure où il est établi que la perception diffère de part et d'autre (Knox et al., 2013). Afin de pallier cette lacune, Schlosser et Gelso ont développé et validé une version de leur questionnaire d'alliance de travail destinée au directeur de thèse (AWAI-A, Schlosser, Gelso, 2005). Complémentaire de la version du doctorant (AWAI-S), les deux questionnaires peuvent être remplis simultanément par les deux membres d'un binôme et mis en correspondance avec différentes variables (personnalité, genre, âge, année de thèse, attitude par rapport à la recherche, etc.). Mais une telle recherche n’a pas encore été menée.

\section{Conclusion}

Les questions de recherches liées à cette relation complexe, déterminante au terme du cursus universitaire, sont innombrables, et les travaux permettant d'y répondre encore trop limités. En particulier, pour le plus grand bénéfice des doctorants autant que celui des encadrants, il importe de clarifier les représentations et les attentes réciproques et de définir les «meilleures pratiques » en fonction des différents contextes. Il est aussi grand temps de proposer aux directeurs de thèse qui le souhaitent de véritables formations pédagogiques à l'encadrement de mémoires et de thèse, encore trop rares dans la majorité des pays francophones.

En effet, le monde de la recherche et l'enseignement supérieur sont en pleine mutation, marqués notamment par l'internationalisation de la production de connaissances, l'obligation croissante de reddition de comptes, l'évaluation de la productivité, la polarisation entre chercheurs « riches » et " pauvres » suivant les financements obtenus, etc. (Leisyte, Dee, 2012 ; Maassen et al., 2012). Elle s'accompagne d'une « réorganisation de la production des connaissances qui ouvre le secteur universitaire aux mécanismes du marché avec, pour conséquence, que les universités deviennent, de fait, des fournisseurs de connaissance dans une économie de la connaissance globale » (Hasselberg, Rider, Waluszewski, 2013). La formation à l'encadrement doctoral devient d'autant plus nécessaire que les directeurs de recherche sont eux-mêmes aux prises avec ces transformations. Ils sont contraints, bon gré mal gré, d'apprendre à s'y adapter, mais ne sont pas encore tous en mesure d'y préparer efficacement les doctorants qu'ils encadrent. 
Dans une conjoncture où le nombre de doctorats accordés chaque année est en augmentation constante dans la plupart des pays de l'OCDE, la qualité de cette préparation est susceptible de jouer un rôle déterminant sur le devenir des jeunes chercheurs, soumis à une concurrence accrue. Le nombre de postes disponibles ne croît pas à la même vitesse que celui des jeunes docteurs. Et il y a peu de chances que la situation s'améliore dans les années à venir.

Tous ces éléments pèsent structurellement sur la relation et la rendent d'autant plus sensible. Pour donner le maximum de chances à chacun de s'épanouir dans sa vie professionnelle à l'issue de la thèse, une piste de plus en plus fréquemment évoquée réside dans l'acquisition et la valorisation de compétences transversales ou soft skills, extrêmement utiles quel que soit le secteur d'exercice. Quelles compétences ? Comment permettre aux étudiants de les développer ? Tels sont les sujets auxquels s'est intéressé, par exemple, le projet ModEs, financé par le programme européen «Lifelong Learning Erasmus » (2009-12)3. Quinze partenaires issus de dix pays différents ont identifié vingtdeux compétences transversales qu'il serait pertinent d'intégrer à nos cursus pour permettre aux universités de continuer à jouer un rôle majeur dans l'innovation. La League of European Research Universities accorde, de la même manière, une grande importance aux personal and professional management skills que les doctorants doivent acquérir durant leur cursus (LERU, 2016).

Sensibiliser également les directeurs de recherche - ou plutôt, dorénavant, les comités de thèse - à la question des soft skills constitue un véritable défi. Certains craindront peut-être de faire ainsi allégeance à un «modèle managérial » de la recherche, qui fait à juste titre l'objet de nombreuses critiques (p. ex., de Gaulejac, 2012). Cependant, chacune des parties aurait tout à gagner à améliorer ses qualités relationnelles, sa créativité, sa capacité d'adaptation ou son éthique professionnelle, pour ne citer que quelques-unes des compétences que le projet ModEs préconise de cultiver chez les étudiants. Évitant une opposition stérile entre les « hordes hétéroclites de Cassandre » et les « néolibéraux » (Hasselberg, Rider, Waluszewski, 2013), il est capital que chaque directeur de recherche se pose la question cruciale soulevée par le sociologue de l'éducation Aaron Pallas il y a déjà plus de quinze ans (2001, cité par Matos, 2013) et prenne la responsabilité d'y apporter des réponses adéquates : Do we want to prepare novice researchers for the world of educational research as it is, or do we want to prepare them for the world as it might become?

\footnotetext{
${ }^{3}$ http://www.modesproject.eu
} 


\section{Bibliographie}

AMUNDSEN C., MCAlPINE L. (2009), « "Learning supervision”: trial by fire », Innovations in Education and Teaching International, vol. 46, $\mathrm{n}^{\circ} 3$, p. 331-342.

APEC (2015), Les jeunes docteurs : profil, parcours, insertion, Les études de l'emploi cadre, $n^{\circ}$ 2015-12. En ligne. URL : http://recherche.cnam.fr/medias/fichier/les-jeunes-docteurs-profil-parcours-insertion-1_1423488756263-pdf.

BAIR C., HAWORTH J. (2005), « Doctoral student attrition and persistence: a meta-synthesis of research » in J. C. SMART (dir.), Higher Education: Handbook of Theory and Research, Dordrecht, Springer, p. 481-534.

BAUMEISTER R. F., LEARY M. R. (1995), «The need to belong: Desire for interpersonal attachments as a fundamental human motivation », Psychological Bulletin, vol.117, $\mathrm{n}^{\circ}$ 3, p. 497-529.

BIBRING E. (1937), « Symposium on the theory of the therapeutic results of psycho-analysis », International Journal of Psycho-Analysis, vol. 18, p. 170-189.

Bravo G., Saint-Mleux J., Dubois M.-F. (2007), « Health Sciences Graduate Students’ Perceptions of the Quality of their Supervision: A Measurement Scale», Canadian Journal of Higher Education, vol. 37, n 2, p. 69-88.

Buckley P. J., Hooley G. J. (1988), « The non-completion of doctoral research in management: symptoms, causes and cures ", Educational Research, vol. 30, n 2, p. 110-120.

ConNell J.P., WellboRn J.G. (1991), « Competence, autonomy, and relatedness: A motivational analysis of self-system processes », in M. R. GunNAR, L.A. SRoufE (dir.), Self processes and development, Hillsdale, NJ, Lawrence Erlbaum Associates, p. 43-77.

De Charms R. (1968), Personal causation; the internal affective determinants of behavior, New York, Academic Press.

DECI E. L. (1975), Instrinsic motivation, New York, Plenum Press.

DECI E. L., RYAN R. M. (1985), Intrinsic motivation and self-determination in human behavior, New York, Plenum Press.

Deci E. L., VAllerand R. J., Pelletier L. G., Ryan R. M. (1991), « Motivation and Education: The Self-Determination Perspective », Educational Psychologist, vol. 26, n 3-4, p. 325-346.

DRENNAN J. (2008), « Postgraduate Research Experience Questionnaire: reliability and factor structure with Master's in Nursing graduates », Journal of Advanced Nursing, vol. $62, \mathrm{n}^{\circ} 4$, p. $487-498$. 
Efstation J. F., Patton M. J., KARdash C. M. (1990), « Measuring the working alliance in counselor supervision », Journal of Counseling Psychology, vol. 37, n 3, p. 322-329.

EMILSSON U. M., JOHNSSON E. (2007). «Supervision of supervisors: on developing supervision in postgraduate education", Higher Education Research e Development, vol. 26, n², p. 163-179.

EtChegoyen R. H., StAgnaro J.-C., WidlÖCher D., Miller J.-A. (2005), Fondements de la technique psychanalytique, trad. O. Begué, Paris, Hermann.

GAULEJAC V. de (2012), La recherche malade du management, Versailles, Éditions Quæ.

GELSO C. J. (1993), « On the making of a scientist-practioner: A theory of research training in professional psychology », Professional Psychology: Research and Practice, vol. $24, \mathrm{n}^{\circ} 4$, p. 468-476.

Gelso C. J., Baumann E. C., Chui H. T., SAVela A. E. (2013), « The making of a scientist-psychotherapist: The research training environment and the psychotherapist ", Psychotherapy, vol. 50, $n^{\circ} 2$, p. 139-149.

GELSO C. J., LENT R. W. (2000), « Scientific training and scholarly productivity: The person, the training environment, and their interaction », in S. D. BROWN, R. W. LENT (dir.), Handbook of counseling psychology, Hoboken, John Wiley \& Sons, p. 109-139.

Gelso C. J., Mallinckrodt B., Judge A. B. (1996), « Research Training Environment, Attitudes toward Research, and Research Self-Efficacy: The Revised Research Training Environment Scale », The Counseling Psychologist, vol. 24, n² 2, p. 304-322.

GeNOUD P. (2003), « Profil des interactions enseignant-élève : traduction, adaptation et validation d'un instrument », L'orientation scolaire et professionnelle, vol. $32, \mathrm{n}^{\circ} 3$, p. 537-552.

GÉRARD L. (2014), Le doctorat : un rite de passage, Paris, Téraèdre.

GoldE C. (2000), «Should I Stay or Should I Go?: Student descriptions of the doctoral attrition process », Review of Higher Education, vol. 23, n 2, p. 119-227.

GoldE C. (2005). « The Role of the Department and Discipline in Doctoral Student Attrition: Lessons from Four Departments ", The Journal of Higher Education, vol. 76, $n^{\circ}$ 6, p. 669-700.

GolDE C. (2007), « Signature Pedagogies in Doctoral Education: Are They Adaptable for the Preparation of Education Researchers? ", Educational Researcher, vol. 36, p. 344351.

Goodman S. B. (2006). "Autonomy and Guidance in Doctoral Advisement Relationships: A Dialectical Study », Humanistic Psychologist, vol. 34, nº 3, p. 201-222. 
Gorga A. M., LeRESCHE J.-P. (dir.) (2015), Disciplines académiques en transformation : entre innovation et résistances, Paris, Éditions des Archives contemporaines.

Grant B., Graham A. (1999), « Naming the Game: reconstructing graduate supervision », Teaching in Higher Education, vol. 4, $n^{\circ}$ 1, p. 77-89.

GreEn J. (2009), « The therapeutic alliance », Child: Care, Health and Development, vol. 35, $\mathrm{n}^{\circ}$ 3, p. 298-301.

GREENSON R. R. (1967), The technique and practice of psychoanalysis, New York, International Universities Press.

GuILlON V. (2013), « L’alliance de travail en psychothérapie, counseling et counseling de carrière », in I. Olry-Louis, V. Guillon, E. LoArer (dir.), Psychologie du conseil en orientation, Bruxelles, De Boeck, p. 129-158.

HAKSEVER A. M., MANiSAli E. (2000), « Assessing supervision requirements of PhD students: The case of construction management and engineering in the UK », European Journal of Engineering Education, vol. 25, n 1, p. 19-32.

HASSELberg Y., Rider S., WALUSZEWSKi A. (2013), «Introduction », in S. Rider, Y. HASSELBERG, A. WALUSZEWSKI (dir.), Transformations in Research, Higher Education and the Academic Market: the Breakdown of Scientific Thought, Dordrecht/New-York, Springer Science/Business Media, p. 1-18.

HeAth T. (2002), «A Quantitative Analysis of PhD Students' Views of Supervision », Higher Education Research \& Development, vol. 21, $\mathrm{n}^{\circ}$ 1, p. 41-53.

HEMER S. R. (2012), «Informality, power and relationships in postgraduate supervision: supervising $\mathrm{PhD}$ candidates over coffee », Higher Education Research er Development, vol. 31, n 6, p. 827-839.

HOllingSWORTH M. A., FASSinger R. E. (2002), "The role of faculty mentors in the research training of counseling psychology doctoral students », Journal of Counseling Psychology, vol. 49, n³, p. 324-330.

Horvath A. O., Greenberg L. S. (1989), « Development and validation of the Working Alliance Inventory », Journal of Counseling Psychology, vol. 36, n 2, p. 223-233.

Knox S., Alan W., JaneceK. J., Pruitt N. T., Fuller. S. L., Hill. C. E. (2011), « Positive and problematic dissertation experiences: The faculty perspective », Counselling Psychology Quarterly, vol. 24, n 1, p. 55-69.

KREMER-HAYON L., WuBBELS T. (1993), « Supervisors' interpersonal behavior and student teachers' satisfaction », in T. WuBBELS, J. LEVY (dir.), Do you know what you look like? Interpersonal relationships in education, London, The Falmer Press, p. 123-135. 
LEARY T. (1957), Interpersonal diagnosis of personality; a functional theory and methodology for personality evaluation, New York, Ronald Press.

LeISYTE L., DEE, J. R. (2012), « Understanding Academic Work in a Changing Institutional Environment. Faculty Autonomy, Productivity, and Identity in Europe and the United States », in J. C. SMART, M. B. PAULSEN (dir.), Higher Education: Handbook of Theory and Research, vol. 27, Dordrecht/New York, Springer, p. 123-206.

LERU (2016), Maintaining a quality culture in doctoral education at research-intensive universities, Advice paper, $n^{\circ} 19$, League of European Research Universities. En ligne. URL : http://www.leru.org/files/publications/LERU_AP19_maintaining_a_quality_culture_in_doctoral_education.pdf.

LitALIEN D., GUAY F. (2015), « Dropout intentions in PhD studies: A comprehensive model based on interpersonal relationships and motivational resources », Contemporary Educational Psychology, vol. 41, p. 218-231.

LOSIER G. (1994), Une analyse motivationnelle de la persévérance aux études avancées, Montréal, Université du Québec à Montréal.

LOVITTS B. E. (2001), Leaving the ivory tower: The causes and consequences of departure from doctoral study, Lanham, Rowman and Littlefield.

Maassen P., Nerland M., Pinheiro R., Stensaker B., Vabø A., Vukasović M. (2012), "Change Dynamics and Higher Education Reforms. Effects in Education, Research, Governance and Academic Profession », in M. VuKasović, P. MAAssen, M. Nerland, R. Pinheiro, B. Stensaker, A. Vabø (dir.), Effects of Higher Education Reforms: Change Dynamics, Rotterdam/Boston, Sense Publishers, p. 1-18.

Mainhard T., VAn Der Rijst R., TARTwijK J. V., Wubbels T. (2009), « A Model for the Supervisor-Doctoral Student Relationship », Higher Education, vol. 58, n³, p. 359-373.

MARKLAND D., ToBin V. J. (2010), « Need support and behavioural regulations for exercise among exercise referral scheme clients: The mediating role of psychological need satisfaction », Psychology of Sport and Exercise, vol. 11, n², p. 91-99.

Matos F. (2013), «PhD and the Manager's Dream: Professionalising the Students, the Degree and the Supervisors? », Journal of Higher Education Policy and Management, vol. 35, nº, p. 626-638.

McAlpine L., AmundSEN C. (2011), Doctoral Education: Research-Based Strategies for Doctoral Students, Supervisors and Administrators, Dordrecht, Springer Science/Business Media.

Moguérou P., Murdoch J., PAul J.-J. (2003), « Les déterminants de l'abandon en thèse : étude à partir de l'enquête Génération 98 du Céreq », 10es Journées D'études Céreq, p. 379-490. 
Overall N., Deane K., Peterson E. (2011), « Promoting doctoral students' research selfefficacy: Combining academic guidance with autonomy support », Higher Education Research e Development, vol. 30, n 6, p. 791-805.

RYAN, R. M. (1995), « Psychological Needs and the Facilitation of Integrative Processes », Journal of Personality, vol. 63, n 3, p. 397-427.

Ryan R. M., DeCi E. L. (2000), «Intrinsic and Extrinsic Motivations: Classic Definitions and New Directions », Contemporary Educational Psychology, vol. 25, n 1, p. 54-67.

SCHLOSSER L. Z., Gelso C. J. (2001), « Measuring the working alliance in advisor-advisee relationships in graduate school », Journal of Counseling Psychology, vol. 48, $\mathrm{n}^{\circ} 2$, p. 157-167.

SCHLOSSER L. Z., Gelso C. J. (2005), « The Advisory Working Alliance Inventory--Advisor Version: Scale Development and Validation», Journal of Counseling Psychology, vol. 52, $n^{\circ} 4$, p. 650-654.

Summers R. F., BARBER J. P. (2010), Psychodynamic therapy: a guide to evidence-based practice, New York, Guilford Press.

TiNTO V. (1993), Leaving college: Rethinking the causes and cures of student attrition, Chicago, The University of Chicago Press.

VACCARo N. (2009), «The Relationship Between Research Self-efficacy, Perceptions of the Research Training Environment and Interest ", in Research in Counselor Education Doctoral Students: An Ex-post-facto, Cross-sectional Correlational Investigation, ProQuest.

VALLERAND R. J., REID G. (1984), "On the causal effects of perceived competence on intrinsic motivation: A test of cognitive evaluation theory ", Journal of Sport Psychology, vol. 6, p. 94-102.

VOYER J.-P. (1992), «Impromptu sur les thèses et leur supervision », Recherche et Formation, vol. 12, p.101-115.

WATZLAWICK P., BAVELAS J. B., JACKSON D. D. (1967), Pragmatics of human communication; a study of interactional patterns, pathologies, and paradoxes, New York, Norton.

Wendler C., Bridgeman B., Markle R., Cline F., Bell N., Mcallister P., Kent J. (2012), Pathways Through Graduate School and into Careers, Princeton, NJ, Educational Testing Service.

White R. W. (1959), « Motivation reconsidered: The concept of competence », Psychological Review, vol. 66, nº 5, p. 297-333. 
WilliAMS G. C., DECI E. L. (1996). «Internalization of biopsychosocial values by medical students: a test of self-determination theory », Journal of Personality and Social Psychology, vol. 70, $\mathrm{n}^{\circ}$ 4, p. 767-779.

Wubbels T., DEN BROK P. J., VAN TARTWijK J. (2006), «An interpersonal perspective on classromm management in secondary classrooms in the Netherlands", in C. EVERTSON, C. WEINSTEIN (dir.), Handbook of classroom management: Research, practice, and contemporary issues, Mahwah (NJ), Lawrence Erlbaum Associates, p. 11611191.

ZUBER-KERRITT O., ROCHE V. (2004), «A constructivist model for evaluating postgraduate supervision: a case study », Quality Assurance in Education, vol. 12, n 2, p. 82-93. 
